\title{
Software Educativo como Método Didáctico en la Enseñanza de las Tablas de Multiplicar
}

\author{
Educational Software as a Didactic Method in the Teaching of Multiplication of \\ Tables
}

Leonardo Marcedonio Piratoba-Gil ${ }^{1}$ \section{RTED
- EDICIÓN: Nesiliencia
aradigmática}

Recibido: 21/junio/2021

OAceptado: $21 /$ septiembre/2021

Publicado: 29/noviembre/2021

$\perp$ Páginas: 53-60

\&aís

${ }^{1}$ Colombia

\section{IIIII Institución}

${ }^{1}$ Universidad Pedagógica

Tecnológica de Colombia

\section{Correo Eletrónico}

1'leonardo.piratoba@uptc.edu.co

\section{(D) ORCID}

${ }^{1}$ https://orcid.org/0000-0001-9557-0994

\section{Citar así: Cf APA / IEEE}

Piratoba-Gil, L. (2021). Software Educativo como Método Didáctico en la Enseñanza de las Tablas de Multiplicar. Revista Tecnológica-Educativa Docentes 2.0, 1(1), 53-60.

https://doi.org/10.37843/rted.v1i1.242

L. Piratoba-Gil, "Software Educativo como Método Didáctico en la Enseñanza de las Tablas de Multiplicar", RTED, vol. 1, n. ${ }^{\circ}$, pp. 53-60, nov. 2021.

\section{Resumen}

Hoy en día las matemáticas siguen siendo un gran obstáculo en la formación de los niños, ya que la observan con temor y desagrado en su proceso de aprendizaje, los profesores en la mayoría de los casos basan sus métodos de enseñanza de manera tradicional y memorística, ocasionando en los estudiantes falta de interés y motivación frente a esta área. Por tal razón, se planteó como objetivo de la presente investigación diseñar un software educativo para ayudar a fortalecer los conceptos de multiplicación en estudiantes de segundo grado de primaria. El estudio se llevó a cabo bajo la metodología investigaciónacción, basado en el modelo pedagógico Escuela Nueva, se realizó el estudio a 15 estudiantes de la escuela de la vereda Santa Ana de la ciudad de Duitama. De igual forma, como instrumentos de recolección de información se aplicó una prueba diagnóstica, una de finalización y una entrevista final, resultados con los cuales se pudo establecer el impacto del software educativo en la enseñanza de las tablas de multiplicar. Los resultados obtenidos dieron muestra de la importancia de la implementación de herramientas como los softwares educativos en la enseñanza de las tablas de multiplicar, en donde se hace esencial poner a la vista de los docentes la utilidad de este material con el propósito de usarlos para mejorar los procesos de enseñanza y aprendizaje en los estudiantes. De esta manera se generó motivación el cual les permitió adquirir habilidades y competencias en matemáticas acorde a como se presenta en el mundo actual.

Palabras clave: Software educativo, método didáctico, tablas de multiplicar.

\section{Abstract}

Today mathematics is still a significant obstacle in the formation of children since they observe it with fear and displeasure in their learning process teachers. In most cases, they base their teaching methods in a traditional and rote way, causing students to lack interest and motivation in this area. For this reason, the objective of this research was to design educational software to help strengthen the concepts of multiplication in second-grade primary school students. The study was carried out under the research-action methodology, based on the Escuela Nueva pedagogical model; the study was carried out on 15 students from the school of the Santa Ana village of the city of Duitama. Similarly, as it applied information gathering instruments, a diagnostic test, a completion test, and a final interview, results established the impact of educational software on the teaching of multiplication tables. The results obtained showed the importance of implementing tools such as educational software in the learning of multiplication tables, where it is essential to show teachers the usefulness of this material to use them to improve teaching and learning processes in students. In this way, motivation was generated, allowing them to acquire skills and competencies in mathematics according to how it is presented today.

Keywords: Educational software, teaching method, multiplication tables. 


\section{Introducción}

Hoy en día las matemáticas siguen siendo un gran obstáculo en la formación de los niños, ya que la observan con temor y desagrado en su proceso de aprendizaje, los profesores en la mayoría de los casos basan sus métodos de enseñanza de manera tradicional y memorística, ocasionando en los estudiantes falta de interés y motivación frente a esta área; debido a esto gracias al avance de la tecnología y a la implementación de las Tecnologías de la Información y Comunicación (TIC). En las Instituciones Educativas se han desarrollado diferentes estrategias con el fin de fortalecer el proceso de enseñanza-aprendizaje, además los métodos de enseñanza van evolucionado gracias a la tecnología; encontrando nuevas formas de aprendizaje en relación con las necesidades e intereses de la actualidad (Bustos \& Román, 2016).

El diseño de software educativos usados en el aula permite crear ambientes divertidos para los estudiantes lo cual conlleva a generar una temática educativa que fortalece los ambientes de aprendizaje, en este caso las tablas de multiplicar. En relación con lo mencionado anteriormente, varios estudios se han enfocado en el aprendizaje de las tablas de multiplicar implementando las nuevas tecnologías como, por ejemplo, investigaciones de Fernández (2007), Isoda \& Olfos (2009), Muñoz (2010), Angulo \& Duarte (2017), Silva, Chiquillo \& Chinome (2017), Tafur, Niño, Martínez, Montero \& Presiga (2016), Agüiño \& Bernal (2017), Carvajal, Escobar \& Usuga (2016), Niño (2018), Becker, McLaughlin, Weber \& Gower (2019), son investigaciones que hacen énfasis en la enseñanza de las tablas de multiplicar usando aprendizajes mediados por las TIC.

En este orden de ideas, continuando con lo mencionado por Muñoz (2010) \& Mounoud (2001) los softwares educativos son herramientas utilizadas con el fin de favorecer los procesos de enseñanza-aprendizaje, los cuales, si son utilizados de forma correcta, permite la creación de ambientes favorables en los estudiantes ocasionando la construcción de conocimiento que se verán reflejados en el mejoramiento académico de ellos, así como su relación con su diario vivir.

Por tal razón, se planteó como objetivo de la investigación diseñar un software educativo que ayude a fortalecer el tema de "multiplicación" en estudiantes de grado segundo de primaria, esta plataforma consta con dos manuales interactivos; el primero presento una guía donde se especifica como está estructurado el software, el segundo muestra el modo de uso del mismo, el software fue creado por autoría propia contando con audio para ayudar a los niños que presentan discapacidades visuales y/o auditivas.

\section{Metodología}

El sistema educativo en la actualidad ha tenido una trasformación desde la aparición del virus denominado COVID-19, lo cual provoco cambios y adaptaciones en las instituciones educativas conllevando a descubrir la utilidad y fortalezas que tiene la tecnología en la educación, así mismo en los contextos investigativos donde se han creado escenarios de investigación en el ámbito de la educación llevándolos a un contexto virtual.

Con base en lo anterior, la presente investigación se basó en la metodología investigación-acción ya que ofrece una salida donde comienza con la crítica a la propia práctica, a través de un proceso de reflexión; profundiza acerca del quehacer pedagógico (Cárdenas-Contreras, 2021, p.75). Este tipo de investigación complementa lo ocurrido entre los agentes de la investigación, por ejemplo, profesores y alumnos, profesores y director, entre otros, además esta investigación se enmarco bajo el modelo pedagógico Escuela Nueva, el cual es:

Un proceso de aprendizaje cooperativo y personalizado centrado en el alumno, la formación de valores y comportamientos democráticos además de poseer el docente un rol como orientador y facilitador, implementando el uso de textos interactivos o guías de aprendizaje para 
lograr así el mejoramiento de la calidad y efectividad del aprendizaje (Colbert, 2006, p.196).

El enfoque de la investigación fue de tipo cualitativo, obteniendo datos descriptivos como palabras textuales de las personas ya sean habladas o escritas, la conducta de los sujetos de la investigación, el entorno en donde se realiza la investigación, por ende, este tipo de investigación:

Es inductiva: Así, los investigadores: Comprenden y desarrollan conceptos partiendo de pautas de los datos, y no recogiendo datos para evaluar hipótesis o teorías preconcebidas. Siguen un diseño de investigación flexible. Comienzan un estudio con interrogantes vagamente formulados. Entiende el contexto y a las personas bajo una perspectiva holística: Las personas, los contextos o los grupos no son reducidos a variables, sino considerados como un todo. Estudia a las personas en el contexto de su pasado y en las situaciones en las que se hallan (Quecedo \& Castaño, 2002, p.7-8).

El estudio se realizó a 15 estudiantes de segundo grado de primaria, bajo la investigación acción $\mathrm{y}$ en el modelo pedagógico de la escuela nueva el cual se aplica actualmente en Colombia, se contó con la opinión y orientación del profesor principal de la asignatura así como los demás profesores de grado segundo, la investigación es de tipo cualitativo con el objetivo de ayudar a los estudiante por medio del software a entender y comprender mejor el tema de multiplicación, se desarrolló un diseño temporal-longitudinal de evolución en grupo donde se examinó a través del tiempo nuestra característica de interés en nuestro grupo a investigar, se recolectó la información a través de una prueba diagnóstica identificando las diferentes falencias presentadas en los estudiantes en este tema llevando a cabo una serie de pasos denominados fases las cuales explican el procedimiento que se llevó a cabo en la investigación, a continuación se mencionan estas fases:

\section{A. Primera Fase: Aplicación de una Prueba Diagnóstica}

En esta fase se diseña una prueba que tiene como finalidad identificar las posibles falencias que tenían los estudiantes sobre el tema "multiplicación", esta prueba consta de 13 puntos entre los cuales se encuentran puntos de unión, representación gráfica, representación numérica y escrita.

\section{B. Segunda Fase: Aplicación y Explicación del Software}

En esta fase se explica las características del software las cuales son: definición de multiplicación, actividades lúdicas, las diferentes tablas de multiplicar, y las técnicas para poder aprender las diferentes tablas (ver Figura 1 y Figura 2).

\section{Tercera fase: Prueba de Verificación de Resultados y Entrevista}

En esta fase se diseñó una prueba con el fin de verificar y determinar si el software ayudó al estudiante a comprender y entender mejor el tema, esta prueba consta de los mismos ítems estipulados en la prueba diagnóstica, pero con diferentes valores, además se realizó una entrevista a tres docentes de grado segundo, con el fin de determinar la metodología usada para enseñar las tablas de multiplicar, así como las posibles dificultades presentadas en los estudiantes a la hora de aprender este tema.

\section{Figura 1}

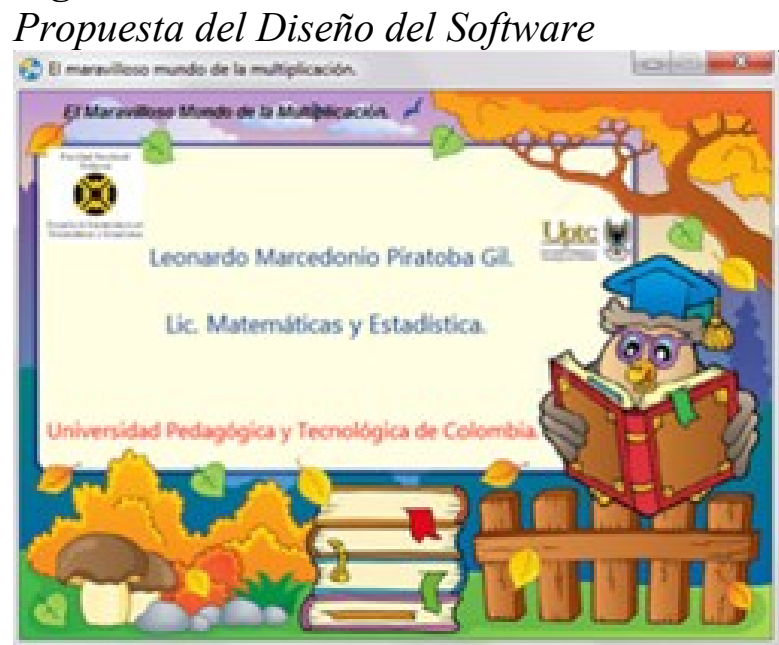

Nota. La Ilustración presenta la portada principal del software educativo, elaboración propia (2020). 


\section{Figura 1}

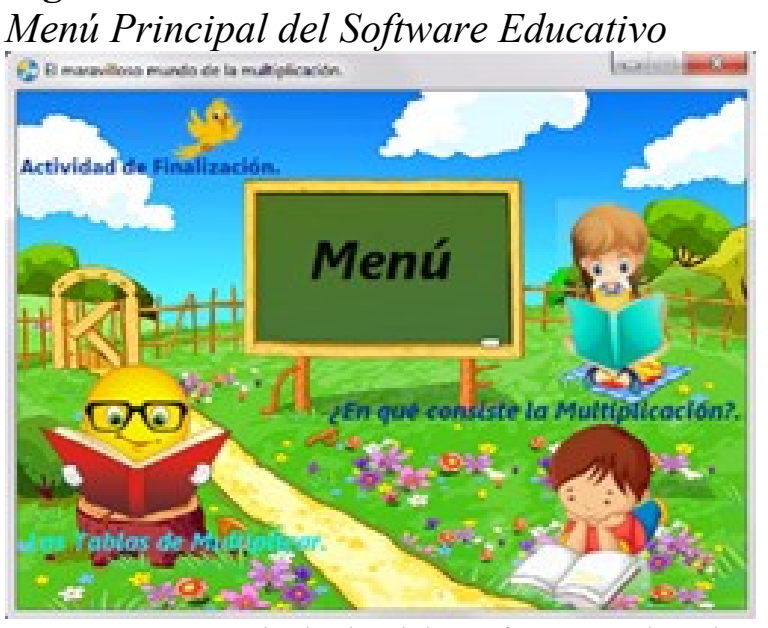

Nota. Menú principal del software educativo, elaboración propia (2020)

Con esto, se pretende utilizar el software educativo para mejorar el proceso de enseñanza de las tablas de multiplicar, logrando que los estudiantes generen una participación y motivación en su propio aprendizaje, conllevándolos a colaborar en todas las fases del proceso de investigación.

\section{Resultados}

Para el análisis de la investigación, se utilizaron los instrumentos de recolección de información mencionados anteriormente, entre los resultados de la prueba diagnóstica se encontraron falencias en las respuestas de los alumnos conllevando a deducir que no comprendían las tablas de multiplicar ocasionando confusión entre ellas y errores al relacionar la multiplicación con su resultado, a partir de esto, la información obtenida se utiliza para determinar el agrado de los estudiantes respecto a su profesor, analizar las dificultades que se presentan dentro del aula, así como el proceso de enseñanza por parte de los docentes.

De lo anterior, se plantearon dos estrategias, la primera se encaminó con la metodología de escuela nueva la cual permitió analizar y cuestionar que actividades incluir en el software educativo con base en la información obtenida en la prueba diagnóstica, la segunda estrategia se realizó teniendo en cuenta las TIC.

Lo anterior permitió crear un análisis categórico teniendo en cuenta las siguientes categorías: la postura del estudiante frente al ánimo de aprender y la atracción, creación del pensamiento lógico matemático por medio del desarrollo de los ejercicios, la Asimilación de los ejercicios, la imaginación para desarrollar los ejercicios y su capacidad de argumentación frente a la respuesta de los ejercicios (ver Tabla 1).

Esto permitió diseñar el software y las actividades que se van a incluir, utilizando el programa Autoplay y Jclic al diseño de las mismas, así como el software Audacity en la creación de audios siendo una guía para los estudiantes con discapacidad visual y/o auditivas, luego se enseñaron las características del software, las herramientas, su uso y se dio paso a la entrega de los manuales tanto a los docentes como a los estudiantes a fin de su correspondiente manipulación.

Los estudiantes dominaron el software, ocasionando alegría y entusiasmo al momento de realizar las actividades planteadas, debido a la estructura agradablemente visual presentada en la plataforma. En el desarrollo de las actividades, se detectó en los estudiantes un aprendizaje favorable originado por la parte multimedia: los gifs y figuras animadas, así como los sonidos que presenta el software.

Al realizar la prueba de verificación se observa una notable mejoría en la disminución de errores que se presentaron en la prueba diagnóstica, lo cual ocasionó una mejor comprensión de las tablas de multiplicar. El análisis de las actividades de esta investigación se presenta en forma de tabla, analizando los elementos importantes a partir de la información obtenida (ver Tabla 2). 
Tabla 1

Análisis de Categorías en los Estudiantes

\begin{tabular}{|c|c|c|c|}
\hline & Categoría & Pre-Propuesta & Pos-Propuesta \\
\hline \multirow[t]{2}{*}{$\begin{array}{l}\text { Postura del } \\
\text { estudiante }\end{array}$} & $\begin{array}{l}\text { Ánimo } \\
\text { aprender }\end{array}$ & $\begin{array}{l}\text { Los estudiantes no } \\
\text { muestran animo ni } \\
\text { interés por aprender } \\
\text { las tablas de } \\
\text { multiplicar. }\end{array}$ & $\begin{array}{l}\text { Las actitudes mostradas por los estudiantes } \\
\text { fueron positivas y agradable generando alegría, } \\
\text { entusiasmo, felicidad, y gusto por la temática } \\
\text { llevándolo a ser más activo, participativo en } \\
\text { cada actividad propuesta en el software } \\
\text { educativo. }\end{array}$ \\
\hline & Atracción & $\begin{array}{lrr}\text { Los } & \text { estudiantes } \\
\text { reflejan que no } & \text { no } \\
\text { sienten deseo ni } \\
\text { atracción } & \text { por } \\
\text { aprender las tablas. }\end{array}$ & $\begin{array}{l}\text { Los estudiantes presentan una nueva faceta en } \\
\text { relación con su actitud ya que el software les } \\
\text { permitió ver la importancia de las tablas de } \\
\text { multiplicar en su vida diaria y a través de los } \\
\text { juegos presentados aprendieron de manera } \\
\text { dinámica esta temática. }\end{array}$ \\
\hline \multirow[t]{4}{*}{$\begin{array}{l}\text { Creación del } \\
\text { pensamiento } \\
\text { Lógico } \\
\text { Matemático }\end{array}$} & Asimilación & $\begin{array}{lr}\text { Los estudiantes } \\
\text { realizan las } \\
\text { multiplicaciones de } \\
\text { forma repetitiva }\end{array}$ & $\begin{array}{l}\text { Los estudiantes muestran su capacidad para } \\
\text { analizar } \\
\text { las partes de la multiplicación, imaginar, } \\
\text { deducir, interpretar y sintetizar la importancia de } \\
\text { la multiplicación con su entorno, además } \\
\text { generar la capacidad para resolver problemas } \\
\text { relacionados a la multiplicación. }\end{array}$ \\
\hline & Ejercicios & $\begin{array}{l}\text { La mayoría de los } \\
\text { estudiantes no } \\
\text { resolvieron la prueba } \\
\text { diagnostica }\end{array}$ & $\begin{array}{l}\text { Los estudiantes reflejaron comodidad en el } \\
\text { aprendizaje de las tablas de multiplicar } \\
\text { expresando agilidad a la hora de resolver los } \\
\text { ejercicios. }\end{array}$ \\
\hline & Imaginación & $\begin{array}{l}\text { No existe } \\
\text { imaginación para } \\
\text { crear otras estrategias } \\
\text { en la solución de } \\
\text { problemas } \\
\text { relacionados con la } \\
\text { multiplicación }\end{array}$ & $\begin{array}{l}\text { Los estudiantes demuestran capacidad para } \\
\text { imaginar y poner en práctica diferentes } \\
\text { estrategias para resolver ejercicios relacionados } \\
\text { con multiplicaciones, así como su capacidad } \\
\text { argumentativa. }\end{array}$ \\
\hline & Argumentación & $\begin{array}{l}\text { Los estudiantes no } \\
\text { presentan } \\
\text { argumentación y no } \\
\text { podían explicar el } \\
\text { porqué de las } \\
\text { respuestas que } \\
\text { eligieron en la prueba } \\
\text { diagnostica }\end{array}$ & $\begin{array}{l}\text { El estudiante genera y es capaz de argumentar } \\
\text { procesos y procedimientos que utilizo para } \\
\text { resolver determinados ejercicios. }\end{array}$ \\
\hline
\end{tabular}

Nota. Categorías de Análisis frente a la interpretación y diseño del software educativo, elaboración propia (2020).

Como respaldo de la investigación, se llevó a cabo una conexión entre tres elementos importantes en esta investigación (Análisis Preliminar, Aplicación de Estrategias, y relación Docente-Alumnos), esta correspondencia la llamaremos "Triada" (ver Figura 3). De acuerdo con la triada se observó que las actividades propuestas llamaron la atención de los estudiantes lo cual genero mucha alegría y motivación al realizar las actividades y las temáticas propuestas en el software.
Los estudiantes realizaron la representación, análisis y composición de cantidades, resolvieron problemas en situaciones multiplicativas, buscaron diferentes métodos de solución, así como estrategias para mejorar su proceso de aprendizaje, logrando una mejor comprensión $\mathrm{y}$ análisis frente a las diferentes situaciones que se presentaron. 


\section{Tabla 2}

Análisis de las Actividades.

\begin{tabular}{l}
\hline Textual \\
\hline Pensamiento \\
Matemático
\end{tabular}

\section{Análisis de la Información}

Matemático

$\mathrm{Al}$ inicio, se presentó dificultad en el manejo del software educativo.

Debido a los procesos de memorización que presentan los estudiantes, se presentan dificultades, pero con las actividades propuestas se apropian de las tablas de multiplicar.

Los estudiantes presentan dificultad en expresar conceptos con sus propias palabras.

Agrado hacia Se evidencia un gran agrado, motivación, interés por parte de los estudiantes al desarrollar las tablas de las actividades propuestas en el software.

multiplicar Los estudiantes presentan dominio del software.

El uso del software educativo permite una mejor recreación de los problemas y además permiten las posibles soluciones.

Estrategia de La estrategia presenta oportunidad en el estudiante con el propósito de motivarlos a enseñanza del desarrollar los ejercicios logrando llamar su atención en toda la actividad.

profesor Esto conlleva a pensar en el uso de la metodología tradicional por parte de los docentes en sus clases lo cual conlleva a los estudiantes a generar un proceso memorístico no practico y mecanizado.

Favorecimiento Los estudiantes se motivan a aprender por medio de diferentes actividades lúdicas.

del uso de las Demuestran facilidad en el manejo del computador, además agilidad en el desarrollo de las TIC

No se presenta distractores, los estudiantes se concentran y atienden a las explicaciones.

Mentalidad Los estudiantes presentaron un cambio de mentalidad, conllevándolos hacer más participativos, activos y a ser protagonistas de las actividades a realizar.

Progreso Los estudiantes trabajaron de manera acorde y efectiva en el software educativo, lograron trabajar con otros compañeros ocasionando un mejor aprendizaje, así como un compañerismos y apoyo entre ellos.

Con base en los conceptos relacionados con las tablas de multiplicar los estudiantes manifestaron interés y agrado por aprender por medio del software, este les permitió cambiar la perspectiva frente a las tablas y así dejar a un lado el temor provocado por las mismas.

El software educativo proporciono nuevas formas de aprendizaje, así como nuevas metodologías a utilizar por parte de los docentes para poder enseñar nuevos conceptos y así mejorar los ambientes de aprendizaje.

Nota. Categorías de Análisis de las actividades planteadas en el software educativo, elaboración propia (2021).

\section{Figura 3}

Triada

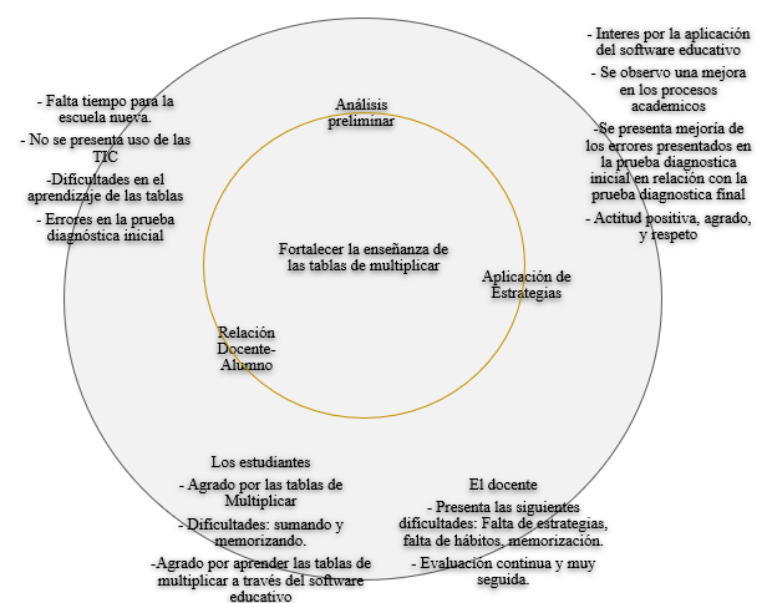

Nota. Relación entre los tres elementos importantes de la investigación, elaboración propia (2021).
En primer lugar, fue necesario identificar las falencias y dificultades en el aprendizaje de los estudiantes a partir del cual los resultados obtenidos con la implementación de la prueba diagnostican reflejaron errores en la interpretación, análisis y respuestas de los ítems propuestos en la prueba, esto evidencia una baja comprensión de las tablas de multiplicar tal como los proceso de multiplicar y además se les dificulta relacionar la multiplicación con su diario vivir.

Por su parte, los resultados obtenidos de la aplicación e implementación del software educativo revelaron la unanimidad de los estudiantes frente al agrado, motivación, emoción y alegría hacia al software educativo, reflejaron una actitud

\section{Conclusiones}


positiva frente a la estructura del software así como a las actividades presentadas en el mismo, de igual modo a las situaciones que estaban encaminadas a la vida cotidiana, y de esta manera se logró en los estudiantes capacidad de análisis, interpretación, comprensión y argumentación frente a situaciones problemas concernientes con la multiplicación, así como un aprendizaje autónomo y divertido de las tablas de multiplicar.

Los resultados obtenidos en la aplicación de la prueba de verificación y en la entrevista, develó una mejoría con respecto a la prueba diagnóstica inicial donde los estudiantes mostraron el uso de diferentes herramientas y métodos en la solución de problemas multiplicativos, así mismo, a pocos estudiantes se les dificultó buscar alternativas de solución frente a diversas situaciones, así como argumentar las respuestas encontradas.

Se puede concluir la necesidad de reconsiderar un cambio en las metodologías, didácticas y pedagogías de enseñanza, en donde en este estudio con el uso y diseño de un software educativo en la enseñanza de las tablas de multiplicar como método didáctico ocasionó en los estudiantes un aprendizaje significativo, el cual les permitió crear nuevos métodos y estrategias de aprendizaje y con ello adquirir habilidades en la solución de problemas concomitantes a conceptos y nociones de multiplicación.

Invito a los docentes a implementar en sus aulas las TIC, y la creación de diferentes plataformas y softwares educativos lo cual genera en los estudiantes aparte de alegría, motivación y además despierta en ellos el deseo de investigar y generar nuevas formas y pautas de aprendizaje.

\section{Reconocimiento}

Agradecimientos del autor al Dr. Publio Suárez Sotomonte director de la Maestría en Educación Matemática de la Universidad Pedagógica y Tecnológica de Colombia y a la profesora MSc. Ruth Paola Gil, docente de la Universidad Pedagógica y Tecnológica de Colombia, gracias por brindarme parte de sus conocimientos y ser guía en la orientación del desarrollo de esta investigación.

\section{Referencias}

Agüiño, M. \& Bernal, Y. (2017). Diseño de un recurso pedagógico para la enseñanza de la multiplicación al integrar GeoGebra en tercer grado de la educación básica primaria. [Tesis de Maestría, Universidad del Valle, Santiago de Cali, Colombia].

https://bibliotecadigital.univalle.edu.co/bitstream/ handle/10893/13594/3469-

0525648.pdf? sequence $=1$ \&isAllowed $=\mathrm{y}$

Angulo, L. \& Duarte, J. (2017). Diseño de una estrategia para favorecer el aprendizaje significativo de la multiplicación en estudiantes de segundo grado de primaria de una institución educativa pública de Bucaramanga, apoyada en el uso de la plataforma Moodle. [Tesis de Maestría, Universidad Cooperativa de Colombia, Bucaramanga, Colombia].

https://repository.ucc.edu.co/bitstream/20.500.124 94/4583/1/PROYECTO\%20DE\%20MAESTRIA. pdf

Becker, A., McLaughlin, T., Weber, K. \& Gower, J. (2019). The effects of copy, cover and compare with and without additional error drill on multiplication fact fluency and accuracy [Article@Efectos del método copiar-tapar-comparar con y sin corrección de errores sobre la fluidez y precisión en las tablas de multiplicar de un estudiante de cuarto curso con dificultades de aprendizaje]. Electronic Journal of Research in Educational Psychology, 7 (2), 747760.

https://www.scopus.com/inward/record.uri?eid=2s2.0-

77951952030\&partnerID $=40 \& \mathrm{md} 5=\mathrm{f} 40 \mathrm{a} 64 \mathrm{~b} 2 \mathrm{dd} 4$ a3232289a000b5a2a37b2

Bustos, A., \& Román, M. (2016). La Importancia de Evaluar la Incorporación y el Uso de las TIC en Educación. Revista Iberoamericana De Evaluación Educativa, 4(2). https://revistas.uam.es/riee/article/view/4452

Cárdenas-Contreras, G. (2021). El mapa conceptual como estrategia pedagógica en el aprendizaje de conceptos disciplinares de Economía. Revista Tecnológica Educativa Docentes 2.0, 11(1), 74-79. https://doi.org/10.37843/rted.v11i1.194

Carvajal, M., Escobar, L. \& Usuga, M. (2016). Aprendamos las tablas de multiplicar y la multiplicación a través de la lúdica y las TIC. [Tesis de Especialización, Fundación Universitaria los Libertadores, Frontino]. https://repository.libertadores.edu.co/bitstream/ha ndle/11371/819/CardonaCarvajalMarjhore.pdf?se quence $=2 \&$ isAllowed $=\mathrm{y}$

Colbert, V. (2006). Mejorar la calidad de la educación en escuelas de escasos recursos. El caso de la Escuela Nueva en Colombia. Revista Colombiana de 


\author{
Educación, $\quad(51), \quad 186-\quad 212$ \\ http://www.redalyc.org:9081/articulo.oa?id $=4136$ \\ 35245
}

Fernández, B. (2007). La Enseñanza De La Multiplicación Aritmética: Una Barrera Epistemológica. Revista Iberoamericana de Educación. 43, 119-130. https://rieoei.org/historico/documentos/RIE43A06 .pdf

Isoda, M., \& Olfo, M. (2009). El Estudio de Clases y las demandas curriculares, La Enseñanza de la Multiplicación. Valparaíso: Ediciones Universitarias de Valparaíso. http://mathinfo.criced.tsukuba.ac.jp/upload/MultiplicationIso daOlfos.pdf

Mounoud, P. (2001). El desarrollo cognitivo del niño: Desde los descubrimientos de Piaget hasta las investigaciones actuales. Contextos Educativos. 4, 53-77.

https://publicaciones.unirioja.es/ojs/index.php/con textos/article/view/486/450

Muñoz, C. (2010). Estrategias didácticas para desarrollar el aprendizaje significativo de las tablas de multiplicar en niños del grado $3-b$ de la Institución Educativa José Holguín Garcés - Sede Ana María De Lloreda. [Tesis de Especialización, Universidad de La Sabana, Bogotá, Colombia]. https://intellectum.unisabana.edu.co/bitstream/han dle/10818/1453/Carmen\%20Lucy\%20Muñoz.pdf? sequence $=1$ \&isAllowed $=\mathrm{y}$

Niño, G. (2018). El software educativo aprende con Erika, en los procesos de aprendizaje de las cuatro operaciones básicas del área de matemáticas, en los estudiantes del grado $3^{\circ}$ de la institución educativa agropecuaria rio sanquianga del municipio de olaya herrera (Nariño). [Tesis de pregrado, Universidad Santo Tomás, Bogotá, Colombia].

https://repository.usta.edu.co/bitstream/handle/116 $34 / 14897 /$ proyecto $\% 20$ con $\% 20$ correcc $\% 20$ del $\% 2$ 0jurado.pdf?sequence $=1 \&$ isAllowed $=\mathrm{y}$

Quecedo, R. \& Castaño, C. (2002). Introducción a la metodología de investigación cualitativa. Revista de Psicodidáctica, (14), 5-39. https://www.redalyc.org/pdf/175/17501402.pdf

Silva, C., Chiquillo, Z. \& Chinome, J. (2017). Scratch. Estrategia didáctica para el aprendizaje de las tablas de multiplicar en escuela nueva. Educación y Ciencia, (20), 43-60. https://revistas.uptc.edu.co/index.php/educacion_y _ciencia/article/view/8897/7352

Tafur, C., Niño, C., Martínez, S., Montero, L. \& Presiga, S. (2016). Software interactivo para el aprendizaje de las tablas de multiplicar de los números enteros positivos. Encuentro Distrital de Educación Matemática. $\quad 344-350$ http://funes.uniandes.edu.co/10187/1/Tafur2016S 